\title{
QT interval prolongation as a biomarker for torsades de pointes and sudden death in drug development
}

\author{
Gregory D. Sides \\ Eli Lilly and Company, Lilly Research Laboratories, Lilly Corporate Center, Indianapolis, IN 46077, USA \\ Tel.: +1 317276 6754; Fax: +1 317277 2025; E-mail: gsides@lilly.com
}

\begin{abstract}
Prolongation of the QT interval on the surface 12-lead electrocardiogram is widely accepted as a biomarker for the potential of a drug to produce torsades de pointes and/or sudden death. Detection of drug-induced prolongation of the QT interval in animals and man is frequently confounded by extrinsic and intrinsic factors that limit the ability to detect a true drug effect. In particular drugs that increase heart rate show an apparent increase in QT interval that confounds assessment of a true drug effect on cardiac ventricular repolarization. The basis for the use of the QT interval as a biomarker will be examined.
\end{abstract}

\section{Introduction}

Drug-induced hepatotoxicity and drug-induced altered cardiac ventricular repolarization are the two most common reasons for withdrawal from market of approved drugs.

The most common manifestation of altered cardiac ventricular repolarization is prolongation of the QT interval, which may progress to torsades de pointes and sudden death. Alternatively, patients may present with torsades or sudden death. Torsades de pointes and sudden death are difficult clinical problems in drug development, because their appearance is unpredictable and rare. Thus, detection of torsades and/or sudden death requires large sample sizes that are typically achieved only after a drug is on the market. Therefore, better methods to assess altered cardiac ventricular repolarization during clinical trials are needed.

The ability of drugs to alter ventricular repolarization was first recognized with the Class I anti-arrhythmic, quinidine. Subsequently Class III anti-arrhythmics were shown to have a similar effect [1]. Class III antiarrhythmic agents block potassium channels, prolong the action potential, increase refractoriness and are used to treat ventricular arrhythmias.
It might be expected that an anti-arrhythmic drug used in patients with heart disease might induce tachydysrhythmias in these patients. However, it was surprising when drugs used to treat non-cardiovascular conditions were reported to prolong ventricular repolarization in patients without apparent heart disease [2, 3].

Subsequently, it has been shown that a number of cardiovascular and non-cardiovascular drugs have the potential to alter cardiac ventricular repolarization (See Table 1) [2,4-14].

Recognition of the potential of non-cardiovascular drugs to prolong the QT interval led to the publication by regulatory agencies of Points to Consider and Guidance documents [15-17].

In 1997, the Committee for Proprietary Medicinal Products (CPMP) of the European Agency for the Evaluation of Medicinal Products (EMEA) issued a document titled "Points to Consider: The assessment of the potential for QT interval prolongation by noncardiovascular medicinal products". The CPMP document recommended in vitro electrophysiological testing, quantitative QT measurement during in vivo preclinical testing, and methods to assess the QT interval in humans to detect altered ventricular repolarization. QT interval increases from baseline in an individual 
Table 1

Examples of drugs associated with QT interval prolongation and torsades de pointes

\begin{tabular}{|c|c|}
\hline Anti-arrhythmics (Class I) & $\begin{array}{l}\text { disopyramide } \\
\text { procainamide } \\
\text { quinidine }\end{array}$ \\
\hline Anti-arrhythmics (Class III) & $\begin{array}{l}\text { amiodarone } \\
\text { dofetilide } \\
\text { sotalol }\end{array}$ \\
\hline Antifungals & $\begin{array}{l}\text { fluconazole } \\
\text { ketoconazole } \\
\text { itraconazole }\end{array}$ \\
\hline Antihistamines & $\begin{array}{l}\text { astemizole } \\
\text { terfenadine }\end{array}$ \\
\hline Antipsychotics & $\begin{array}{l}\text { chlorpromazine } \\
\text { haloperidol } \\
\text { risperidone } \\
\text { sertindole }\end{array}$ \\
\hline GI motility agents & $\begin{array}{l}\text { cisapride } \\
\text { mosapride }\end{array}$ \\
\hline Macrolide antibiotics & $\begin{array}{l}\text { erythromycin } \\
\text { clarithromycin }\end{array}$ \\
\hline Quinolone antibiotics & $\begin{array}{l}\text { grepafloxacin } \\
\text { sparfloxacin }\end{array}$ \\
\hline
\end{tabular}

patient of $<30 \mathrm{msec}$ are unlikely to represent risk of inducing torsade, $30-60 \mathrm{msec}$ are more likely to represent a drug effect and $>60 \mathrm{msec}$ raise clear concerns about the potential risk of inducing torsade. The recommendations in the CPMP document have created a new paradigm for drug development where assessment for altered ventricular repolarization is now an integral component of every new drug development program.

In 2001, the Therapeutic Products Directorate (TPD) of the Bureau of Pharmaceutical Assessment of Health Canada issued a draft guidance document titled "Assessment of the QT prolongation potential of nonantiarrhythmic drugs". The Health Canada document parallels the CPMP document in its' recommendations.

In 2002, the US Food and Drug Administration (FDA) is expected to issue a draft guidance on QT prolongation. The Japan Ministry of Health Labor and Welfare has not issued a written QT guidance document.

QT interval prolongation has resulted in the withdrawal from market or restricted sales in one or more countries for several drugs [18].

\section{Mechanism of prolongation}

Development of drug-induced QT interval prolongation and torsades de pointes occurs with drugs that block the delayed rectifier potassium channel, IKr [19].
The IKr channel plays an important role in repolarization and in the normal rate-dependent shortening of the action potential. The channel is composed of proteins encoded for by two genes. The human ether-relateda-go-go (HERG) gene encodes for the pore-forming HERG protein and the KCNE2 gene encodes for the accessory protein, MinK-related peptide 1 (MiRP1) [2022].

With cardiac myocyte repolarization, potassium ions move out of the cell. Drug-induced prolongation of cardiac repolarization is primarily due to slowing the movement of potassium ions out of the cell by blocking the efflux of potassium through the $\mathrm{IKr}$ channel.

Drug access to the channel is from the cytoplasm. The drug enters the channel vestibule when the activation gate is open. With subsequent depolarization, the drug is trapped in the voltage-gated channel. The large size of the IKr channel vestibule and the amino acid constituents of the pore may contribute to the increased susceptibility of the channel to blockade compared to other cardiac ion channels [23,24].

With decrease in the outward movement of potassium ions from the myocyte, the action potential of the cell is prolonged.

The mechanism for the generation of torsades is not known with certainty. An increase in dispersion of repolarization, early after depolarizations, and re-entry within the ventricular wall may be involved $[18,25]$.

The vast majority of patients who present with druginduced QT prolongation do not have congenital mutations of HERG or KCNE2. However, the presence of a forme fruste of a long QT syndrome may be present in some patients with drug-induced QT prolongation [22].

\section{Preclinical assessment}

As awareness of the ability of drugs to induce QT interval prolongation increased, it became apparent that detection of the problem was not occurring in the preclinical assessment or in clinical trials. This awareness led to the development of better methods to detect altered ventricular repolarization.

The International Conference on Harmonization (ICH) has published a draft consensus guideline regarding safety pharmacology studies for assessing the potential for delayed ventricular repolarization (QT interval prolongation) by human pharmaceuticals [17].

Virtually all drugs are now screened in in vitro ionic current assays, particularly in a heterologous expression system. The HERG gene is stably transfected 
into a cell culture of human embryonic kidney cells (HEK 293). A concentration response curve is generated. The inhibitory concentration (IC50) is compared to the effective concentration (EC50) to determine a cardiac safety index [26]. Clinically relevant blockade is generally accepted to occur when $\geqslant 20 \%$ inhibition of the $\mathrm{I}_{\mathrm{Kr}}$ channel is observed at anticipated therapeutic plasma or myocardial tissue concentrations of parent drug or metabolite(s). Thus, it is important to know the protein binding of parent and metabolite(s) in order to interpret the in vitro $\mathrm{IKr}$ data.

In addition, in vitro action potential duration assays are recommended in the $\mathrm{ICH}$ document. Action potentials represent the integrated activities of the multiple ion channels.

In vivo repeat-dose toxicology studies in dog or nonhuman primate are now conducted with quantitative, rather than qualitative, assessment for QT interval prolongation.

\section{Clinical assessment}

Electrocardiograms should be obtained from healthy subjects and patients in Phase I and II trials. Sufficient electrocardiograms should be collected to determine whether there is a dose-response relationship between drug dose and QT interval duration. The clinical plan should also include careful assessment for an effect of gender, age and metabolism. Drugs metabolized oxidatively through cytochrome $\mathrm{P} 450$ should be carefully assessed for altered ventricular repolarization.

For drugs that do not block the IKr channel at clinically relevant drug concentrations in the HERG assay and do not prolong the QT interval in dog or monkey, electrocardiograms should be obtained from a minimum of 100 subjects in clinical trials [15].

For drugs that block the HERG channel in vitro or prolong the QT interval in animals, electrocardiograms should be obtained from a minimum of 200 subjects [15].

If $\mathrm{I}_{\mathrm{Kr}}$ channel blockade or QT prolongation in animal toxicology studies occurs, then electrocardiograms will also likely need to be collected in Phase III trials.

In Phase I trials, a time course of electrocardiograms should be obtained prior to and during study drug administration. In Phase II trials, several electrocardiograms should be obtained prior to and during study drug administration, particularly at the time of anticipated peak drug concentrations for parent and any relevant metabolites.

\section{Measurement of the QT interval}

Cardiac ventricular repolarization is assessed in clinical trials by measuring the QT interval on a 12-lead surface electrocardiogram. The interval is measured at the time of collection by a computer algorithm embedded in many electrocardiogram machines. Subsequently, the computer-measured interval is "overread" by a cardiologist or technician. The human-measured interval is obtained by manual measurement with handheld calipers or with computer assistance using a digitizing pad.

The offset of the QT interval may be difficult to ascertain due to baseline artifact, the presence of a $U$ wave or the presence of an abnormal $\mathrm{T}$ wave.

Accurate and reproducible assessment of the QT interval is made more difficult by the lack of a "gold standard" method of measurement. Thus, no method can claim to be more accurate. Methods should be compared for their reproducibility.

The most common method for manual measurement of the QT interval is the tangent method [27]. In general the QT interval is measured in lead II. The U wave is commonly not included in the measurement. The offset of the T wave is the intersection of a tangent line with the iso-electric line. The tangent line is drawn along the maximum downslope of the T wave. The QT interval is measured in three beats and averaged.

The computerized measurement employed by GE Marquette creates a median waveform complex for each lead composed of all beats acquired from each lead during a 10 -second acquisition period. A signal-average complex is constructed and the QT interval is measured. Computer-measurement enhances reproducibility. The computer measurement from the waveform complex is generally longer than the manually measured QT interval.

Without a standardized procedure and process, there can be considerable intra- and inter-observer variability in the measurement. It is important to understand the method for determination of the offset of the $\mathrm{T}$ wave and the leads in which the QT interval was measured. The goal is to measure the QT interval within $\pm 5 \mathrm{msec}$. However, the width of the stylus ink on the electrocardiogram is approximately $10 \mathrm{msec}$ making the accuracy goal difficult. In one study, $36.9 \%$ of measurements differed between observers by 10 to $20 \mathrm{msec}$ and $29.5 \%$ differed by $30 \mathrm{msec}$ or greater [28].

The parameter of greatest interest in drug development is the change in QT interval from a predrug baseline to the time of anticipated maximal drug concen- 
tration. Therefore, reproducibility and accuracy in QT measurement are equally important.

The combination of a computer-measured interval followed by cardiologist overread may provide the most reproducible and accurate measurement. All computermeasured QT intervals should be overread by an experienced electrocardiographer. One approach is to accept the computer-measured interval, unless there is baseline artifact, an abnormal $\mathrm{T}$ wave or the presence of a U wave that interfere with the computer's ability to accurately determine the offset of the $T$ wave. In such instances the cardiologist should manually measure the QT interval.

\section{Physiological variation of the QT interval}

In addition to measurement variation, there is also physiological variation in the QT interval. The QT interval varies with heart rate, autonomic tone, time of day and presence/absence of cardiovascular disease.

The QT interval is inversely related to heart rate; the slower the heart rate the longer the QT interval. The QT interval is "corrected" for heart rate using one or more equations to minimize the correlation between the QT and RR intervals. The most common correction method is Bazett's equation $\left(\mathrm{QTcB}=\mathrm{QT} / \mathrm{RR}^{0.5}\right)$. However, Bazett's equation was derived from a small sample of healthy subjects and does not represent a clinical trial population. In addition, Bazett's equation does not minimize the correlation sufficiently, particularly for drugs that increase heart rate.

Drugs that increase heart rate can appear to prolong the QTcB interval, even for drugs that only increase heart rate by a few beats per minute. Therefore, accurate assessment of QTc requires better correction methods. Several methods are currently in development.

The mean QT interval is longer at baseline in patients with cardiovascular disease $(417 \mathrm{msec})$ compared to healthy subjects $(407 \mathrm{msec})$. The likelihood that a patient with cardiovascular disease will have a prolonged QT interval due to underlying disease is also greater when compared to healthy subjects. In addition, the increase in mean QT interval with terfenadine administration is greater in patients with cardiovascular disease (12 msec) compared to healthy subjects $(6 \mathrm{msec})$. The spontaneous variability in QT interval was greater than the drug-induced increase in QT interval. The spontaneous variability may confound the ability to detect a true drug effect [2].
The QT interval is prolonged in patients with newly diagnosed or established type II diabetes mellitus compared to nondiabetic subjects. Blood pressure and left ventricular mass were the main determinants of QT interval duration in both patient populations. Coronary artery disease in newly diagnosed diabetes was also independently related to QT interval [29].

Assessment for QT interval prolongation is also confounded by the diurnal variation in the QT interval, with the longest QT interval during sleep [30]. Thus, the time of day should be considered when standardizing protocol design.

\section{Effect of gender on the QT interval}

Women are at greater risk than men of drug-induced QT interval prolongation. The difference in risk may be due to several factors. Women have a longer QT interval than men. In addition hormonal differences may contribute to the disparity.

There is a gradual increase in the QT interval in women as they age. In men there is shortening of the QT interval at the onset of puberty, then a gradual increase in the QT interval with aging [31].

In rabbits, sex hormones prolong the QT interval and downregulate potassium channel expression in the heart. Sex hormones may be important in modulating cardiac repolarization [32].

In healthy men and women, mean baseline QT intervals were longer in women $(407 \pm 7 \mathrm{msec})$ than men (395 $\pm 9 \mathrm{msec})$. Quinidine caused greater QT prolongation in women than in men at equivalent serum concentrations. The slope of the change in QTc interval from baseline to the serum concentration of quinidine was $44 \%$ greater in women than men; the difference was statistically significant [33].

Women had a 3-fold greater odds of developing torsades de pointes than men in sotalol clinical trials where the anti-arrhythmic was evaluated for the treatment of supraventricular and ventricular arrhythmias [34].

Of 332 patients with torsades de pointes receiving cardiovascular drugs that prolong cardiac repolarization, $70 \%$ of reported cases were women. Females were statistically significantly more prevalent when cases of torsades de pointes were analyzed by individual drug [35].

The degree of drug-induced QT prolongation in response to administration of the anti-arrhythmic, ibutilide, varied with the menstrual cycle phase. The greatest increase in QTc occurred in the first half of the menstrual cycle [36]. 


\section{Summary}

There is a need for an evidence-based approach to acquiring, analyzing and interpreting QT interval data from clinical pharmacology studies and clinical trials. QT interval prolongation has an established presence as a biomarker for torsades de pointes and sudden death. However, given the difficulties in accurately determining the offset of the $\mathrm{T}$ wave, better methods are needed to predict the potential for a new drug to alter ventricular repolarization.

\section{References}

[1] M. Zehender, S. Hohnloser and H. Just, QT-interval prolonging drugs: mechanisms and clinical relevance of their arrhythmogenic hazards, Cardiovasc Drugs Ther 5(2) (1991), 515530.

[2] C.M. Pratt, S. Ruberg, J. Morganroth, B. McNutt, J. Woodward and S. Harris et al., Dose-response relation between terfenadine (Seldane) and the QTc interval on the scalar electrocardiogram: distinguishing a drug effect from spontaneous variability, Am Heart J 131(3) (1996), 472-480.

[3] P.K. Honig, D.C. Wortham, K. Zamani, D.P. Conner, J.C. Mullin and L.R. Cantilena, Terfenadine-ketoconazole interaction. Pharmacokinetic and electrocardiographic consequences, JAMA 269(12) (1993), 1513-1518.

[4] C. Torp-Pedersen, M. Moller, P.E. Bloch-Thomsen, L. Kober, E. Sandoe and K. Egstrup et al., Dofetilide in patients with congestive heart failure and left ventricular dysfunction. Danish Investigations of Arrhythmia and Mortality on Dofetilide Study Group, N Engl J Med 341(12) (1999), 857-865.

[5] K. Chiba, A. Sugiyama, Y. Satoh, H. Shiina and K. Hashimoto, Proarrhythmic effects of fluoroquinolone antibacterial agents: in vivo effects as physiologic substrate for torsades, Toxicol Appl Pharmacol 169(1) (2000), 8-16.

[6] Y. Satoh, A. Sugiyama, K. Chiba, K. Tamura and K. Hashimoto, QT-prolonging effects of sparfloxacin, a fluoroquinolone antibiotic, assessed in the in vivo canine model with monophasic action potential monitoring, J Cardiovasc Pharmacol 36(4) (2000), 510-515.

[7] J.L. Demolis, A. Charransol, C. Funck-Brentano and P. Jaillon, Effects of a single oral dose of sparfloxacin on ventricular repolarization in healthy volunteers, Br J Clin Pharmacol 41(6) (1996), 499-503.

[8] H. Dupont, J.F. Timsit, B. Souweine, B. Gachot, M. Wolff and B. Regnier, Torsades de pointe probably related to sparfloxacin, Eur J Clin Microbiol Infect Dis 15(4) (1996), 350-351.

[9] J. Vitola, J. Vukanovic and D.M. Roden, Cisapride-induced torsades de pointes, J Cardiovasc Electrophysiol 9(10) (1998), 1109-1113.

[10] R. Ohki, M. Takahashi, O. Mizuno, H. Fujikawa, T. Mitsuhashi and T. Katsuki et al., Torsades de pointes ventricular tachycardia induced by mosapride and flecainide in the presence of hypokalemia, Pacing Clin Electrophysiol 24(1) (2001), 119-121.

[11] L. Carlsson, G.J. Amos, B. Andersson, L. Drews, G. Duker and G. Wadstedt, Electrophysiological characterization of the prokinetic agents cisapride and mosapride in vivo and in vitro: implications for proarrhythmic potential? J Pharmacol Exp Ther 282(1) (1997), 220-227.

[12] A.H. Glassman and J.T. Jr. Bigger, Antipsychotic Drugs: Prolonged QTc Interval, Torsade de Pointes, and Sudden Death, Am J Psychiatry 158(11) (2001), 1774-1782.

[13] W. Haverkamp, A. Martinez-Rubio, C. Hief, A. Lammers, S. Muhlenkamp and T. Wichter et al., Efficacy and safety of d,1sotalol in patients with ventricular tachycardia and in survivors of cardiac arrest, J Am Coll Cardiol 30(2) (1997), 487-495.

[14] J.L. Bauman, R.A. Bauernfeind, J.V. Hoff, B. Strasberg, S. Swiryn and K.M. Rosen, Torsade de pointes due to quinidine: observations in 31 patients, Am Heart J 107(3) (1984), 425430 .

[15] Committee for Proprietary Medicinal Products (CPMP), $\mathrm{Hu}-$ man Medicines Evaluation Unit. The European Agency for the Evaluation of Medicinal Products (EMEA). Points to consider: The assessment of the potential for QT interval prolongation by non-cardiovascular medicinal products, 1997, CPMP/98/696, www.ifpma.org/ich1.html.

[16] Therapeutic Products Directorate, Bureau of Pharmaceutical Assessment. Health Canada. Draft guidance document: Assessment of the QT prolongation potential of nonantiarrhythmic drugs, 2001, www.hc-sc.gc.ca.

[17] International Conference on Harmonization, Draft Consensus Guideline. Step 2. Safety pharmacology studies for assessing the potential for delayed ventricular repolarization (QT interval prolongation) by human pharmaceuticals, 7 Feb 2002, www.ifpma.org/ich1.html.

[18] W. Haverkamp, G. Breithardt, A.J. Camm, M.J. Janse, M.R. Rosen and C. Antzelevitch et al., The potential for QT prolongation and pro-arrhythmia by non-anti- arrhythmic drugs: clinical and regulatory implications. Report on a Policy Conference of the European Society of Cardiology, Cardiovasc Res 47(2) (2000), 219-233.

[19] M.C. Sanguinetti and N.K. Jurkiewicz, Two components of cardiac delayed rectifier $\mathrm{K}+$ current. Differential sensitivity to block by class III antiarrhythmic agents, J Gen Physiol 96(1) (1990), 195-215.

[20] M.C. Sanguinetti, C. Jiang, M.E. Curran and M.T. Keating, A mechanistic link between an inherited and an acquired cardiac arrhythmia: HERG encodes the IKr potassium channel, Cell 81(2) (1995), 299-307.

[21] J. Warmke, R. Drysdale and B. Ganetzky, A distinct potassium channel polypeptide encoded by the Drosophila eag locus, Science 252(5012) (1991), 1560-1562.

[22] G.W. Abbott, F. Sesti, I. Splawski, M.E. Buck, M.H. Lehmann and K.W. Timothy et al., MiRP1 forms IKr potassium channels with HERG and is associated with cardiac arrhythmia, Cell 97(2) (1999), 175-187.

[23] J.S. Mitcheson, J. Chen and M.C. Sanguinetti, Trapping of a methanesulfonanilide by closure of the HERG potassium channel activation gate, J Gen Physiol 115(3) (2000), 229240 .

[24] J.S. Mitcheson, J. Chen, M. Lin, C. Culberson and M.C. Sanguinetti, A structural basis for drug-induced long QT syndrome, Proc Natl Acad Sci USA 97(22) (2000), 12329-12333.

[25] C. Antzelevitch, Z.Q. Sun, Z.Q. Zhang and G.X. Yan, Cellular and ionic mechanisms underlying erythromycin-induced long QT intervals and torsade de pointes, J Am Coll Cardiol 28(7) (1996), 1836-1848.

[26] I. Cavero, M. Mestre, J.M. Guillon and W. Crumb, Drugs that prolong QT interval as an unwanted effect: assessing 
their likelihood of inducing hazardous cardiac dysrhythmias, Expert Opin Pharmacother 1(5) (2000), 947-973.

[27] B. Surawicz and S.B. Knoebel, Long QT: good, bad or indifferent? J Am Coll Cardiol 4(2) (1984), 398-413.

[28] P.A. Gorman, J.B. Calatayud, S. Abraham and C.A. Caceres, Observer variation in interpretation of the electrocardiogram, Medical Annals of the District of Columbia 33(3) (1964), 9799.

[29] A. Festa, R. Jr. D’Agostino, P. Rautaharju, L. Mykkanen and S.M. Haffner, Relation of systemic blood pressure, left ventricular mass, insulin sensitivity, and coronary artery disease to QT interval duration in nondiabetic and type 2 diabetic subjects, Am J Cardiol 86(10) (2000), 1117-1122.

[30] A. Jr. Garson, How to measure the QT interval - what is normal? Am J Cardiol 72(6) (1993), 14B-16B.

[31] P.M. Rautaharju, S.H. Zhou, S. Wong, H.P. Calhoun, G.S. Berenson and R. Prineas et al., Sex differences in the evolution of the electrocardiographic QT interval with age, Can J
Cardiol 8(7) (1992), 690-695.

[32] M.D. Drici, T.R. Burklow, V. Haridasse, R.I. Glazer and R.L. Woosley, Sex hormones prolong the QT interval and downregulate potassium channel expression in the rabbit heart, $\mathrm{Cir}$ culation 94(6) (1996), 1471-1474.

[33] R.E. Benton, M. Sale, D.A. Flockhart and R.L. Woosley, Greater quinidine-induced QTc interval prolongation in women, Clin Pharmacol Ther 67(4) (2000), 413-418.

[34] M.H. Lehmann, S. Hardy, D. Archibald, B. Quart and D.J. MacNeil, Sex difference in risk of torsade de pointes with d,1-sotalol, Circulation 94(10) (1996), 2535-2541.

[35] R.R. Makkar, B.S. Fromm, R.T. Steinman, M.D. Meissner and M.H. Lehmann, Female gender as a risk factor for torsades de pointes associated with cardiovascular drugs, JAMA 270(21) (1993), 2590-2597.

[36] I. Rodriguez, M.J. Kilborn, X.K. Liu, J.C. Pezzullo and R.L. Woosley RL, Drug-induced QT prolongation in women during the menstrual cycle, JAMA 285(10) (2001), 1322-1326. 


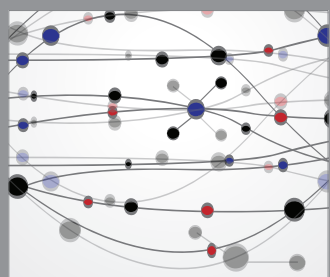

The Scientific World Journal
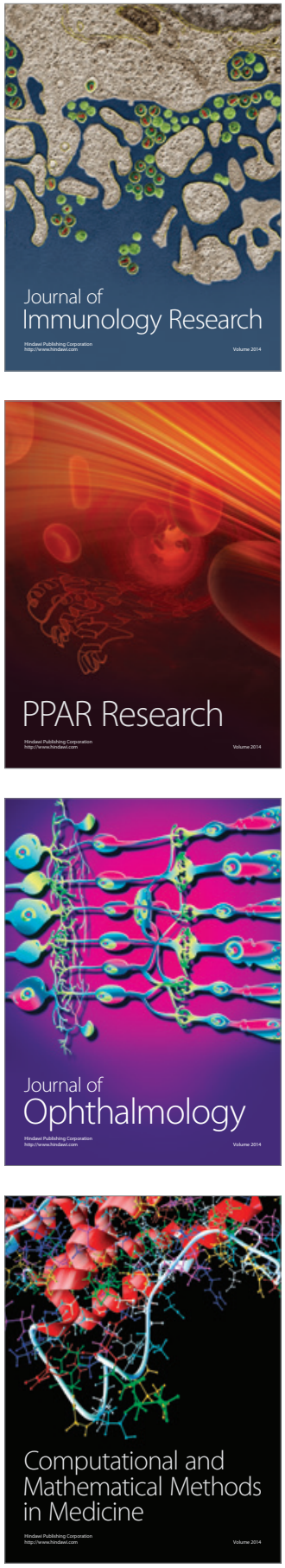

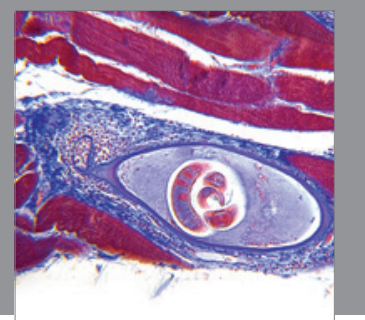

Gastroenterology

Research and Practice
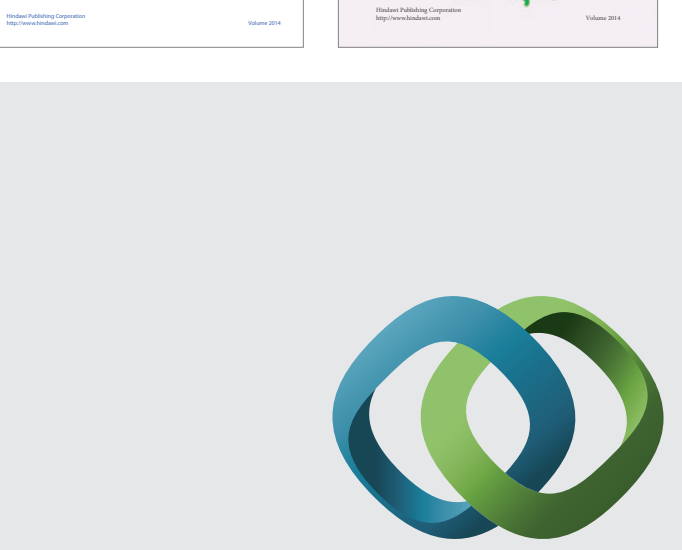

\section{Hindawi}

Submit your manuscripts at

http://www.hindawi.com
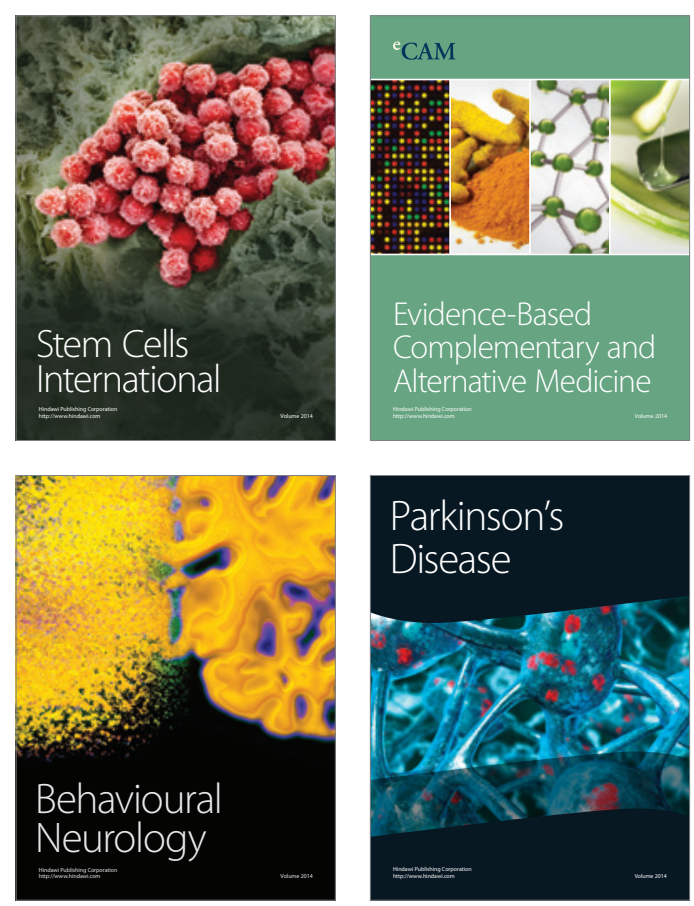

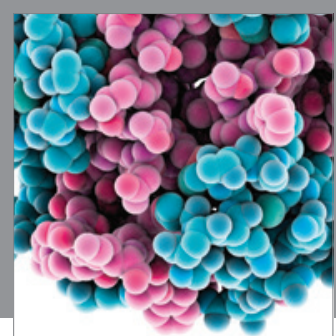

Journal of
Diabetes Research

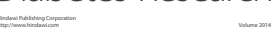

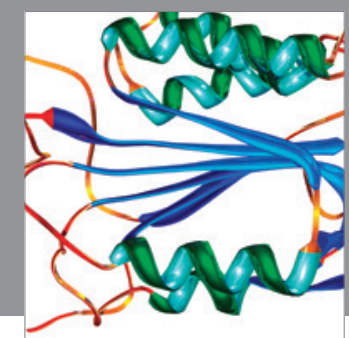

Disease Markers
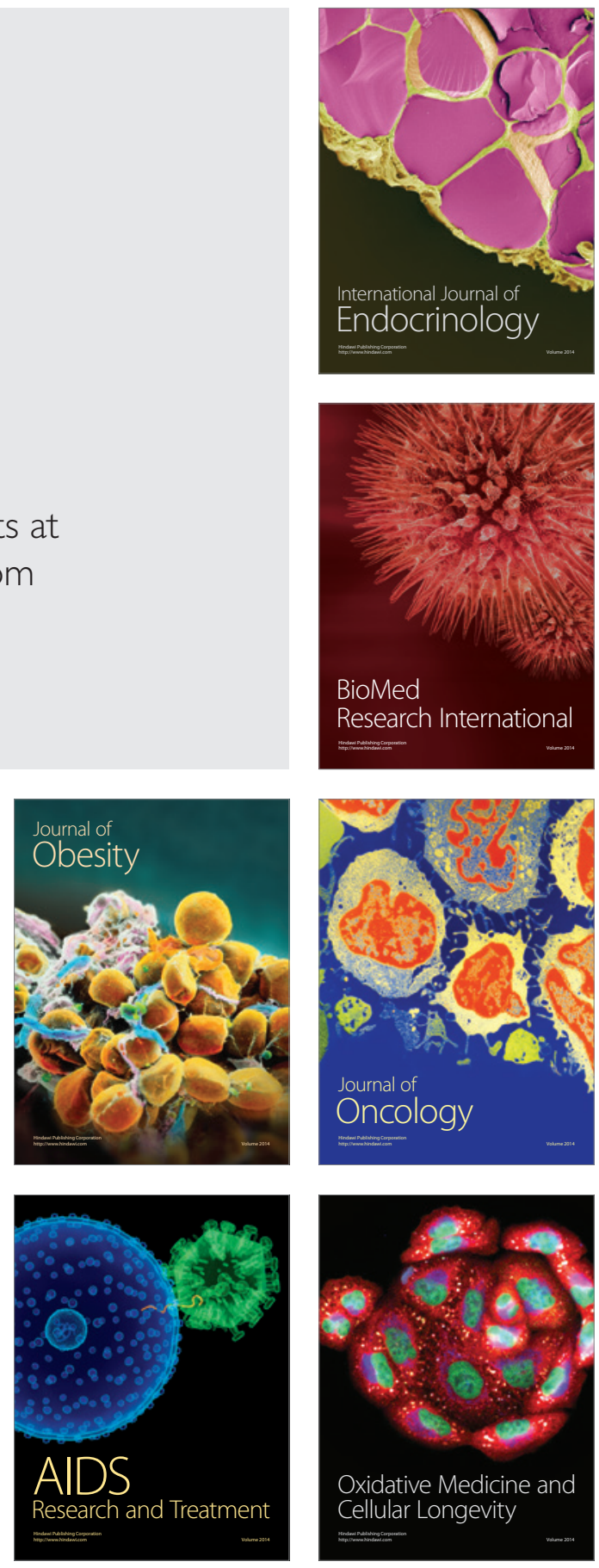